\title{
Thickness and Surface Measurement of Transparent Thin-Film Layers using White Light Scanning Interferometry Combined with Reflectometry
}

\author{
Taeyong Jo, KwangRak Kim, SeongRyong Kim*, and HeuiJae Pahk \\ School of Mechanical and Aerospace Engineering, Seoul National University, Seoul 151-741, Korea
}

(Received March 21, 2014 : revised May 12, 2014 : accepted May 12, 2014)

\begin{abstract}
Surface profiling and film thickness measurement play an important role for inspection. White light interferometry is widely used for engineering surfaces profiling, but its applications are limited primarily to opaque surfaces with relatively simple optical reflection behavior. The conventional bucket algorithm had given inaccurate surface profiles because of the phase error that occurs when a thin-film exists on the top of the surface. Recently, reflectometry and white light scanning interferometry were combined to measure the film thickness and surface profile. These techniques, however, have found that many local minima exist, so it is necessary to make proper initial guesses to reach the global minimum quickly. In this paper we propose combing reflectometry and white light scanning interferometry to measure the thin-film thickness and surface profile. The key idea is to divide the measurement into two states; reflectometry mode and interferometry mode to obtain the thickness and profile separately. Interferogram modeling, which considers transparent thin-film, was proposed to determine parameters such as height and thickness.

With the proposed method, the ambiguity in determining the thickness and the surface has been eliminated. Standard thickness specimens were measured using the proposed method. Multi-layered film measurement results were compared with AFM measurement results. The comparison showed that surface profile and thin-film thickness can be measured successfully through the proposed method.
\end{abstract}

Keywords : Transparent thin film, Thickness, Interferometry, Reflectometry, Interferogram modeling OCIS codes : (120.0120) Instrumentation, measurement, and metrology; (120.3180) Interferometry

\section{INTRODUCTION}

Thin films have been increasingly used in the optics, semiconductors and material industries to provide specific functions to the coated devices. Effective measurement of the profile and the film thickness are important to achieve special functions. To measure the thickness and surface, optical methods such as white-light interferometry and reflectometry have been widely used. Recently, white light scanning interferometry has extended its application from the surface measurement to the thin film thickness measurement. However, due to the phase distorting effect of the transparent thin-film, it is very difficult to measure a transparent thin-film on the top of the 3-D surface by conventional metrology.

Reflectometry is more popular in industries for its fast throughput. Reflectometry is a well-established and powerful technique for measuring film thickness $[1,2]$. It measures reflectance for different wavelengths to calculate thickness. But, this technique can only determine the film thickness and it is limited to only one point detection. Recently, volumetric thickness profile has been measured using a CCD camera [3].

To measure the 3-D profile of a transparent surface, a white light interferometry technique which gets spectrally resolved analysis by Fourier transform was proposed [3-4]. It determines both surface profile and film thickness in the spectral domain. Kim's method extracted thickness information from the interferogram. It has the merit that it does need additional spectral units, but spectral information usually is not enough for calculating the thickness in a practical measurement. And it has the limitation of spectral resolution, which is most important in calculating thickness. To compensate for the ambiguity in spectral analysis, reflectometry and

\footnotetext{
*Corresponding author: srkim1303@gmail.com

Color versions of one or more of the figures in this paper are available online.
} 
spectrally resolved white light interferometry were combined $[5,6]$. But this method was too sensitive to the detection of the peak signal. Modeling based researches also had been proposed recently [7-10]. And several researchers have used different techniques to measure the thin film thickness and height accurately [11-20]. However, there still remains ambiguity in detecting surface height due to the thin film effect. They had practical limitations of uncertainty and measurement speed. To overcome those problems we have combined a spectrometer into an interferometry system. As a result, the proposed method can gather more accurate and highly resolved spectral information for thickness calculation.

In this paper we combine the white light scanning interferometry and reflectometry for determination of film thickness and surface profile. The key idea is to divide the measurement into two states, reflectometry mode and interferometry mode, to get the two unknowns of thickness and surface profile separately. The experiment was performed in two steps. First the film thickness was determined in reflectometry mode. For the next step, the thickness and surface profile are calculated by white light scanning interferometry mode using the thickness value which is determined by reflectometry mode. It saves calculation time for least-square fitting because it does not need an initial guess for the thickness value. For reflectometry mode, a normal objective lens and spectrometer were used to measure the thickness of the specimen. For interferometry mode, an interferometric lens (mirau type) and CCD sensor were used to measure the film thickness and surface profile. The shape and position of an interferogram varies depending on several parameters such as the height and thickness of the specimen. And it can be modeled using the theory of interferometry and reflectometry. The optimum model provides the surface profile and the thinfilm thickness simultaneously. The final thin-film and surface profile were measured by obtaining the best matching model using the non-linear least square fitting. For the interferometry mode, we considered the phase changing effect of the thin-film on the top of the surface for accurate and simultaneous measurements of the film thickness and surface profile.

By separating the thickness measurement from the surface profile measurement using reflectometry, we enhanced the accuracy of both surface profiling and thickness measurement. The most important advantage of the proposed technique is the elimination of the ambiguity in determination of thickness and surface by separating measurement of thickness and surface profile.

\section{EXPERIMENTAL SETUP}

The experimental setup is shown in Fig. 1. A vertical movement in the $\mathrm{z}$ direction was implemented using the piezoelectric actuator toward the test surface located in the $\mathrm{x}-\mathrm{y}$ plane. The test surface is observed through objective (Nikon ELWD 10X/20X/50X) lenses and an interferometric (Nikon 20X/50X Mirau type) objective. The light source is a halogen lamp with a broad and continuous spectrum.

The setup can be used both for reflectometry measurement mode or interferometry measurement mode. When it uses a normal objective lens and spectrometer, it does not allow the light reflected on the reference surface to reach the spectrometer because a normal objective lens does not have a reference mirror inside. So, under this condition, the spectrometer records interference between the top and bottom surface of the film in the specimen. This is known as reflectometry mode. When the interferometric lens and the CCD camera were used, the light reflected on the reference surface. The reflection interfered with the test surface beam. Now the CCD camera records the interference signal between the test and reference signals. This mode is known as white light interferometery mode. In this mode, the interferogram gives information about the thin film thickness $d$ and surface height $h$ simultaneously.

For the measurement of reflectometry mode, the spectrometer (Ocean Optics QE65000; 1024 pixels), which has a detecting range from $200 \mathrm{~nm}$ to $1100 \mathrm{~nm}$, was used. The spectrometer is aligned at the center of the camera so that the light reflected from the center of the specimen can enter the spectrometer. By implementing a normal objective lens and $50 \mu \mathrm{m}$ optical fiber (Ocean Optics) between the beam splitter and the spectrometer, a $2.5 \mu \mathrm{m}$ spot for reflectometry measurement was implemented.

For the measurement of interferometry mode, the interferometric objective was fitted with a PZT. The control voltage to shift the PZT for phase shifting was produced by a Micom with PID control. The camera (Vieworks VG264, $250 \mathrm{fps}$ ) gives an 8-bit digital output. The interferograms were transferred to the $\mathrm{PC}$ through an image acquisition board.

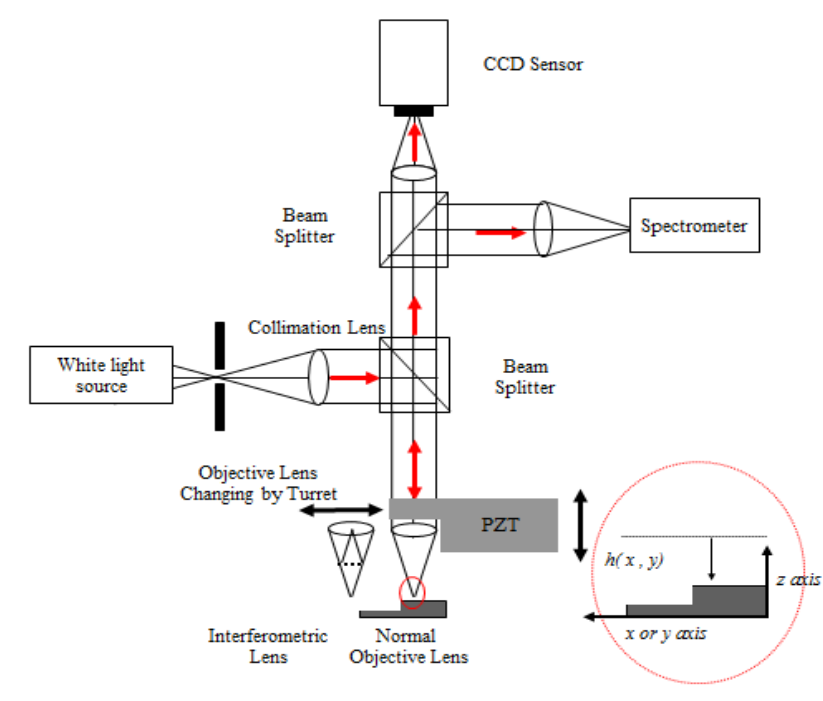

FIG. 1. Experimental setup. 


\section{PRINCIPLE}

\subsection{Multiple Reflection}

When incident light is upon a surface with a transparent thin-film layer, multiple reflection takes place, as illustrated in Fig. 2. If we take account of the multiple reflections inside the transparent thin film layer, the parameter $R$ (total reflection coefficient) can be derived as Eq. (1) [1]

$$
\begin{aligned}
& R=\frac{r_{01}+r_{12} \exp (-j 2 \beta)}{1+r_{01} r_{12} \exp (-j 2 \beta)} \\
& \beta=2 \pi \frac{d}{\lambda} N_{1} \cos \left(\theta_{1}\right)
\end{aligned}
$$

where $r_{01}$ and $r_{12}$ represents the Fresnel reflection coefficients of the top and the bottom boundaries of the film, respectively. $\beta$ denotes the phase change of the reflected wave as it traverses the thin-film. $\beta$ can be expressed explicitly as Eq. (2) where $\theta_{1}$ is the propagation angle of the incident beam and $d$ and $N_{1}$ are the thickness and the refractive index of the thin-film layer, respectively. The parameter $R$ (the reflection coefficient of the surface) is a complex number which can be expressed in the standard form of

$$
R=a+b i
$$

where $a$ and $b$ are real numbers. Then the total phase difference of the reflected beam relative to the incident beam is determined as

$$
\psi=\tan ^{-1}(b / a)
$$

\subsection{Interferogram Modeling}

Considering the reflection of the specimen, interference signals can be expressed as a sum of reference light and objective light as,

$$
\begin{aligned}
& \mathrm{E}_{\text {ref }}(\mathrm{z}, \mathrm{k})=\mathrm{E}_{\mathrm{i}(\mathrm{k})} \rho(1-\rho)\left|R_{r e f}(k)\right| * \mathrm{e}^{-\mathrm{i}\left(2 \mathrm{kl} 1-\psi_{\text {ref }}\right)} \\
& \mathrm{E}_{\text {obj }}(\mathrm{z}, \mathrm{k})=\mathrm{E}_{\mathrm{i}(\mathrm{k})} \rho(1-\rho)\left|R_{o b j}(k)\right| * \mathrm{e}^{-\mathrm{i}\left(2 \mathrm{k}(1+\mathrm{h}-\mathrm{z})-\psi_{\text {obj }}\right)}
\end{aligned}
$$

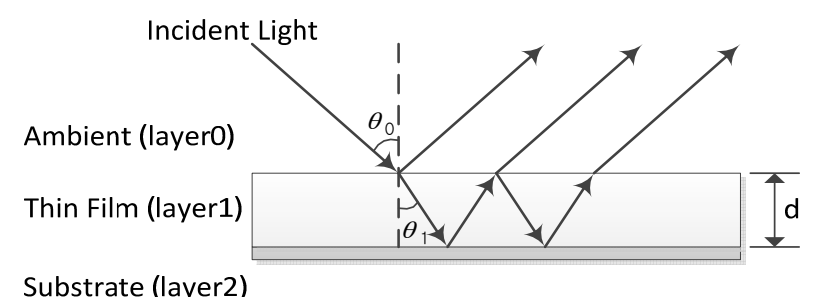

Substrate (layer2)

FIG. 2. Multiple reflections in thin film.

$$
\begin{aligned}
& \varphi(z, k)=\left(E_{r e f}+E_{o b j}\right) \overline{\left(E_{r e f}+E_{o b j}\right)} \\
& \Delta \psi=\psi_{r e f}-\psi_{o b j}
\end{aligned}
$$

where, $\mathrm{z}$ is the position of PZT, $\mathrm{k}$ is the wavenumber, $E_{\text {ref }}(z, k)$ is the wave equation of reference light, $E_{o b j}(z, k)$ is the wave equation of measurement light. $\rho$ is the reflection coefficient of the beam splitter, $\left|R_{r e f}(k)\right|$ is the reflection coefficient of the reference light, $\left|R_{o b j}(k)\right|$ is the reflection coefficient of the objective. $l$ is the length difference between the reference mirror and the objective lens, $h$ is the height of the sample, $\mathrm{z}$ is the position of scanning optics. $\psi_{\text {ref }}$ and $\psi_{o b j}$ are the initial phase in reference mirror and sample. Here we need to remember that $\psi_{o b j}$ is the function of the film thickness and wavenumber.

Considering incoherent superposition of monochromatic lights, the interference intensity sampled from a surface point $(\mathrm{x}, \mathrm{y})$ of height $\mathrm{h}$ is generally described as the integral of the effective wave number from $k_{1}$ to $k_{2}$

$$
\varphi(z)=\int_{k 1}^{k 2} I_{0}[1+\gamma \cos (2 k(h-z)-\Delta \Psi)]^{*} F(k) d k
$$

where $\gamma$ denotes the modulation amplitude and $F(k)$ and $d k$ are, respectively, the central wave number and the bandwidth of the white-light source. In Eq. (9), the integral is related to the broad spectral distribution of the source light. To simplify the analysis, the numerical aperture of the objective is assumed to be small enough for approximating $\cos \left(\theta_{0}\right)=1$ and $\sin \left(\theta_{0}\right)=0 . \Delta \Psi$ is the initial phase difference between $\psi_{\text {ref and }} \psi_{o b j}$. Calculating $\psi_{o b j}$ using Eq.(4) and substituting $\Delta \Psi$ in Eq. (9) by Eq. (8), for each wavelength from $k_{1}$ to $k_{2}$, an interferogram considering the phase change due to the thin-film can be modeled. The spectral distribution of the light source is considered from $400 \mathrm{~nm}$ to $800 \mathrm{~nm}$. For comparison, the interferogram shape without film was modeled, as shown in Fig. 3(a) and the interferogram with a $408 \mathrm{~nm} \mathrm{SiO} 2$ film on $\mathrm{Si}$, as shown in Fig. 3(b).

In the case of Fig. 3(a), the psi of the sample does not vary because there is no thin-film on the top layer. In the case of 3(b), the interference of the top layer is affected by the signal from the bottom surface. As the thin film gets thinner, the interference signal of the bottom surface affects the interference signal of the top surface.

\subsection{Non-linear Least Square Fitting}

In reflectometry mode, after the data acquisition process is completed, a regression method, such as the LevenbergMarquardt non-linear fitting algorithm, is used for searching the best-matching theoretical reflectance. The aim of the fitting process is to find the thin film thickness that minimizes the difference between the measured reflectance 


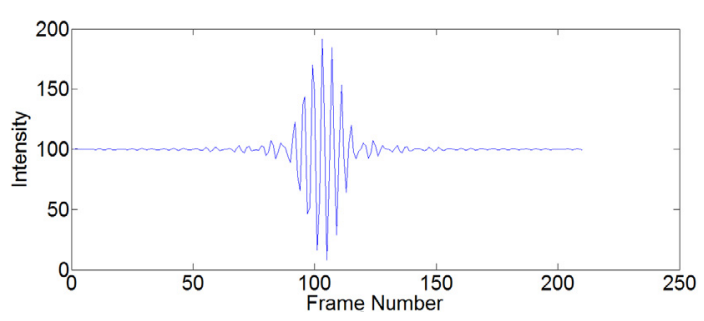

(a)

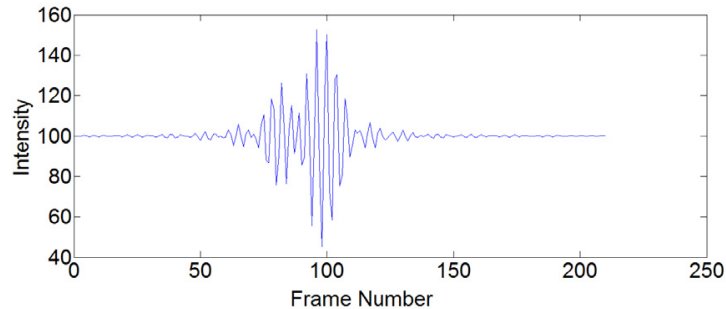

(b)

FIG. 3. Modeled interferograms of (a) no film (b) $\mathrm{SiO}_{2} 408 \mathrm{~nm}$ film on Si.

and the theoretically calculated reflectance. In order to evaluate the difference, the error, e(d), in Eq.(10) is used. The difference is squared and integrated in wavelength range. As the error gets close to zero, the theoretical reflectance gets more similar to the measured reflectance.

$$
e(d)=\int_{-\infty}^{\infty}\left[R_{\text {experiment }}(d)-R_{\text {theory }}(d)\right]^{2} d \lambda
$$

In interferometry mode, surface height $h$ and thickness $d$ can be determined using Non-Linear Least Square Fitting. In this work, measured $\varphi_{\text {measured }}$ denotes the actual data gathered from the system and $\varphi_{\text {theory }}$ is the modeled interferogram result. An error function is defined as the sum of squared deviations.

$$
\chi(d, h)=\sum_{z=\text { FrameStart }}^{\text {FrameEnd }}\left(\varphi_{\text {measured }}(z)-\varphi_{\text {theory }}(z ; d, h)\right)^{2}
$$

The optimum model shows the surface profile $h$ and thin-film thickness $d$ simultaneously. The phase component in the Eq. (9) turns out to be highly nonlinear with respect to the unknowns; thus an appropriate multidimensional nonlinear least-squares technique with fast convergence capability is necessary. In searching for the true values of $h$ and $d$, we found that many local minima exist, so it was necessary to make proper initial guesses for these values to reach the global minimum quickly. For determination of thickness and surface in interferometry mode, thickness data measured by reflectometry mode was applied as the initial thickness value $d$. Finally the best matching interferogram gives height and thickness information of the measured specimen.

\section{EXPERIMENTAL RESULTS}

The experiment was performed in two steps. First, thin film thickness was determined in reflectometry mode and then the film thickness and the surface profile was calculated by interferometry mode. For interferometry measurement, the thickness value determined by reflectometry mode was used as the initial thickness value $d$.

\subsection{Reflectometry Mode Result}

A regression method like Levenberg-Marquardt nonlinear fitting algorithm is used for searching for the best-matching theoretical reflectance. The aim of the fitting process is finding the thickness of thin film which minimizes the difference between measured reflectance and theoretically calculated reflectance. The procedure of finding the best matching model is shown in Fig. 4.

Using the reflectometry mode, the proposed reflectance and phase modeling was verified. $\mathrm{SiO}_{2}$ film of $408 \mathrm{~nm}$ thickness was deposited on a $\mathrm{Si}$ substrate by NIST; NIST 400-100, CRADA, CN-1364, 19984. Figure 5 shows the fitting result between the measured reflectance signal and

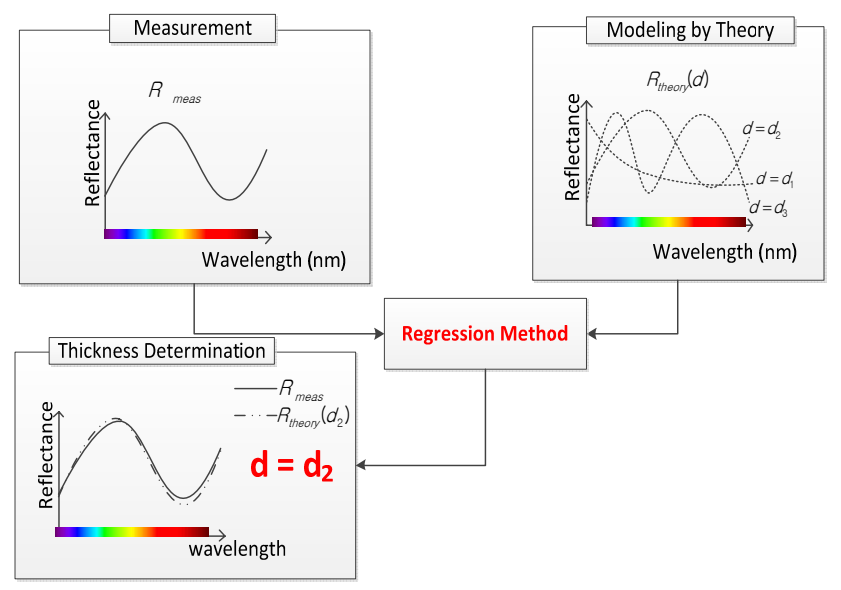

FIG. 4. Procedure of regression algorithm.

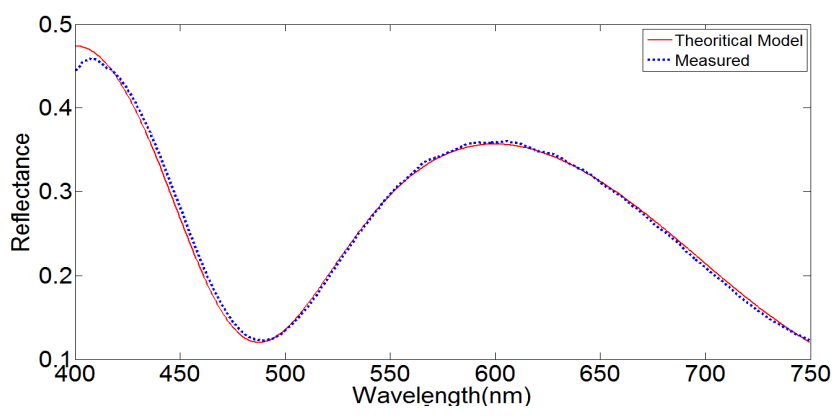

FIG. 5. Fitting of experimental and modeled reflectances for $\mathrm{SiO}_{2} 408 \mathrm{~nm}$ on $\mathrm{Si}$. 
modeled reflectance signal. We searched for the optimal thickness of the film thickness ' $d$ ' that would minimize the error function e(d) in Eq. (10). Figure 5 shows the fitted reflectance.

\subsection{Interferometry Mode Result}

The thickness ' $d$ ' measured in reflectometry mode was set as the initial thickness value of interferometry mode. Fig. 6 shows the fitting result of the sample. Matching results of the actual interferogram and the modeled signal were plotted. Thickness of the interferometry mode was analyzed by averaging $10 * 10$ pixels at the center of the specimen.

To verify the effectiveness of the interferogram modeling, reference specimens which have $\mathrm{SiO}_{2}$ film layers on silicon have been analyzed; NIST reference specimen for $408 \mathrm{~nm}$ (NIST 400-100, CRADA, CN-1364, 19984) and Mikropack-ID0076 specimen for the others (from $40 \mathrm{~nm}$ to $1000 \mathrm{~nm})$.

To verify the performance of the proposed technique, samples of thickness lower than $200 \mathrm{~nm}$ also have been investigated. Fig. 7 shows the comparison of the true value and measured thickness value. When the thickness is lower than $150 \mathrm{~nm}$, correct measurement result is not guaranteed; it shows the limitation of the proposed method. The reason for the limitation is when the deposited film gets thinner than $150 \mathrm{~nm}$, thicknesses can no longer be distinguished from one to the other because the phase change within the thin-film layer becomes very small regardless of wave number when thickness $d$ is smaller than $150 \mathrm{~nm}$.

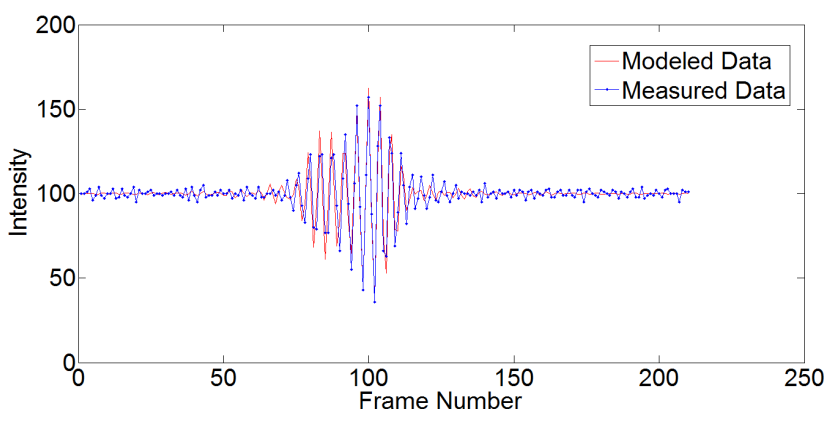

FIG. 6. Experimental and fitted interferograms for $\mathrm{SiO}_{2} 408 \mathrm{~nm}$ on Si.

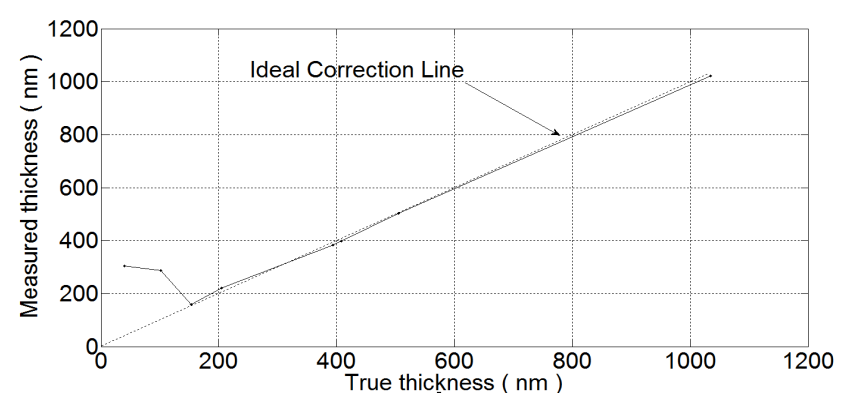

FIG. 7. Comparison of true and measured thickness values.
For verifying proposed reflectometry, we measured samples in Table 1 using three objectives with different magnification. The test result indicates that the maximum deviation reaches; $0.6 \%$ when the thickness value is in the range $350 \mathrm{~nm}-1100 \mathrm{~nm}$. It shows enhanced thickness measurement accuracy compared with previous research; $3 \%[4]$

The thin-film-transistor (TFT) supporting structure, which is a very important structure in the Liquid Crystal Display panel illustrated in Fig. 8, was measured by using the proposed method for industrial application. The sample has two layers from the bottom. The first layer is relatively thick with opaque material and the second layer consists of transparent thin film. These kinds of samples could be measured to evaluate the performance of the proposed method. Table 3 shows the measured results for

TABLE 1. Comparison of sample thicknesses measured by two methods (unit : nm)

\begin{tabular}{c|c|c|c}
\hline \hline & True Value & Reflectometry & $\begin{array}{c}\text { Proposed } \\
\text { Interferferometry }\end{array}$ \\
\hline Sample1 & 42 & 41 & 312 \\
\hline Sample2 & 83 & 84 & 298 \\
\hline Sample3 & 168 & 169 & 172 \\
\hline Sample4 & 205 & 206 & 221 \\
\hline Sample5 & 394 & 401 & 384 \\
\hline Sample6 & 408 & 409 & 398 \\
\hline Sample7 & 505 & 507 & 503 \\
\hline Sample8 & 1035 & 1040 & 1022 \\
\hline
\end{tabular}

\begin{tabular}{l|l|} 
Transparent \\
Opaque \\
Substrate
\end{tabular}

FIG. 8. Structure of specimen to verify simultaneous measurement performance using the interferometry mode.

TABLE 2. Comparison of sample thicknesses measured by reflectometry mode with three objectives (unit : nm)

\begin{tabular}{c|c|c|c}
\hline \hline & $10 \mathrm{X}$ & $20 \mathrm{X}$ & $50 \mathrm{X}$ \\
\hline Sample1 & 42 & 41 & 42 \\
\hline Sample2 & 85 & 84 & 86 \\
\hline Sample3 & 169 & 169 & 168 \\
\hline Sample4 & 206 & 206 & 206 \\
\hline Sample5 & 401 & 401 & 399 \\
\hline Sample6 & 409 & 409 & 408 \\
\hline Sample7 & 506 & 507 & 504 \\
\hline Sample8 & 1039 & 1040 & 1038 \\
\hline
\end{tabular}


the sample shown in Fig. 8. AFM measurement results are also provided in Table 3 for comparison. The conventional bucket algorithm by Larkin [17] gave a different value from the true value due to the thin-film effect on the top of the surface. On the other hand, the proposed method showed a maximum difference of $9 \mathrm{~nm}$ from the AFM results. Accuracy of the conventional method is not guaranteed when transparent film exists; $22.1 \%$ error (Table 3). On the other hand the proposed method showed enhanced accuracy; $0.7 \%$. It shows enhanced surface measurement accuracy compared with previous research; $3 \%[4]$.

In searching for the true values of $h$ and $d$ we have found that many local minima exist, so it is necessary to make good initial guesses at these values to reach the global minimum. Figure 9 shows a series of calculation for Eq.(11) that we performed to verify the convergence of the least-squares technique for Sample 1 in Table 3. 20 times iteration has been performed for each calculation to perform a feasibility test. The computation result reveals that global minimum is attained within 20 iterations for proper initial guess. However, when the inappropriate initial guess value had been given, correct convergence is not guaranteed; it depends a great deal on the initial guesses of $d$. If we do not apply initial value $d$ in the equation, we cannot expect correct result for both $h$ and $d$. Also it took some tens of seconds to determine the thickness and height of the single point without proper initial guess value. For these reasons, the measurement range of the thickness was set to be in range from $(d$ -100) $\mathrm{nm}$ to $(d+100) \mathrm{nm}$. It gives a huge decrease in calculation time because it does not need an initial guess for the thickness. When we applied the proposed method, it took less than 3 seconds to analyze the target area of

TABLE 3. Comparison between the conventional method and the proposed method (unit: $\mathrm{nm}$ )

\begin{tabular}{l|c|c|c}
\hline \hline & AFM & $\begin{array}{c}\text { Conventional } \\
\text { Interferometry }\end{array}$ & Proposed method \\
\hline Sample1 & 1113 & 1359 & 1120 \\
\hline Sample2 & 1702 & 1467 & 1693 \\
\hline Sample3 & 2106 & 1874 & 2097 \\
\hline
\end{tabular}

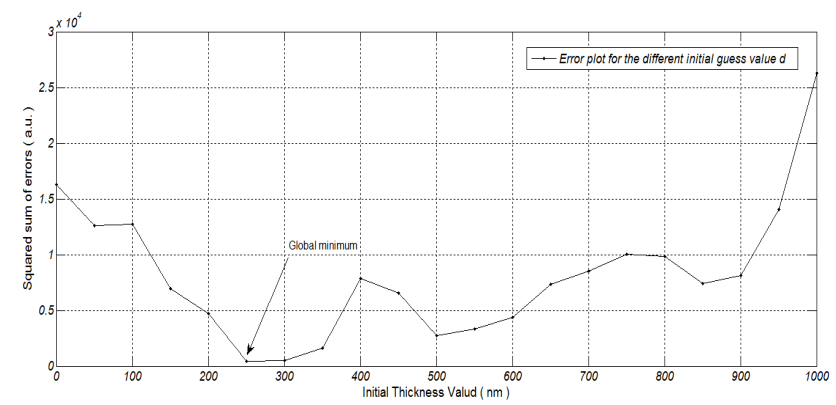

FIG. 9. Error plot for the different initial value.
$100 * 100$ pixels including nonlinear least square fitting. As the calculation time has been decreased, 3-D surface with transparent thin-film can be measured in practice.

For verifying proposed methods, we measured samples of Table 2 using two objectives with different magnification. The test result indicates that the maximum deviation reaches; $0.5 \%$ when the magnification varies from $20 \mathrm{X}$ to 50X (Table 4).

The profile of the sample was compared with the AFM measurement result, as shown in Fig. 10.

The comparison showed fairly good matching profile. Finally, a 3-D surface profile measured using the proposed algorithm is shown in Fig. 11.

\section{CONCLUSION}

White light scanning interferometry is a well-established technique in surface profiling applications. The resolution

TABLE 4. Comparison of sample heights measured by interferometry mode with three objectives (unit: $\mathrm{nm}$ )

\begin{tabular}{c|c|c}
\hline \hline & $20 \mathrm{X}$ & $50 \mathrm{X}$ \\
\hline Sample1 & 1120 & 1124 \\
\hline Sample2 & 1693 & 1692 \\
\hline Sample3 & 2097 & 2099 \\
\hline
\end{tabular}

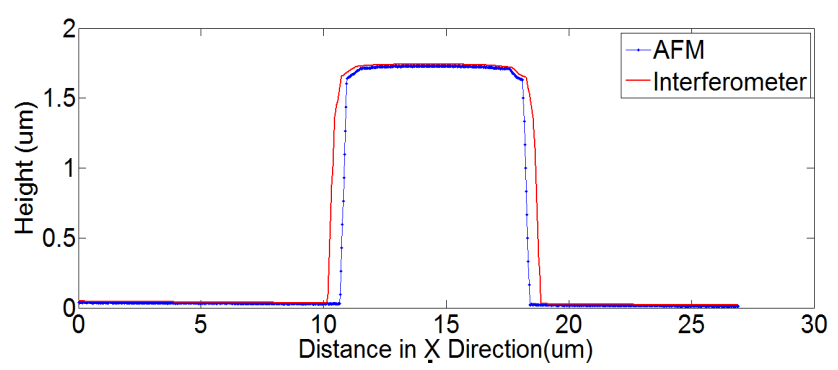

FIG. 10. Comparison between interferometry- and AFMmeasured profiles.

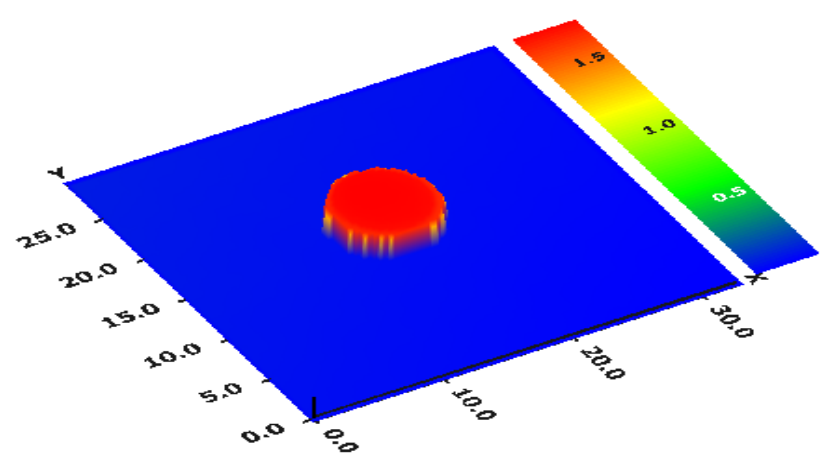

FIG. 11. 9 3-D measurement of TFT-support by proposed method. 
in this technique is of the order of a nanometer. But when a transparent thin film exists, the reflection signals from both the top and bottom surfaces of the film will interfere with the reference signal. At the same time, the multiple reflection signals between the two film surfaces will also interfere with the others. Effective separation of the interference signals from each other is the key to achieving a successful measurement. In this paper, measurement of the surface profile and thin-film thickness by interferometry combined with reflectometry was proposed. The key idea is to divide the measurement into two states using reflectometry mode and interferometry mode to obtain the two unknowns separately; thickness $d$ and height $h$. The final thin-film surface profile information is measured by obtaining the best matching model using the non-linear least square fitting. The proposed method gave the proper initial guess for the accurate measurement and it saves calculation time in finding the most adequate interferogram. It took less than 3 seconds to analyze target area of $100 * 100$ pixels.

The maximum difference between the measured height and the reference height was more than $250 \mathrm{~nm}$ under the conventional bucket algorithm, but the difference was reduced to $16 \mathrm{~nm}$ under the proposed method. Accuracy has been enhanced from $22.1 \%$ to $0.7 \%$ for height measurement using the proposed method. The modeling method was more effective when it was used for a thin-film $(<1 \mu \mathrm{m})$ because the phase distortion of the thin-film gets worse as the thin film gets thinner. The AFM profile showed a fairly good matching result with the profile of the proposed method. This technique is available not only probing the thickness point by point but also providing complete volumetric film profiles digitized in three dimensions. At present, this measurement technique provides nanometer resolutions of the vertical height when the transparent film thickness exceeds $150 \mathrm{~nm}$. The limitation occurs when the deposited film gets thinner than $150 \mathrm{~nm}$. The reason is that the interferogram can no longer be distinguished from one to the other because the phase change within the thin-film layer becomes very small regardless of the wave number when thickness $d$ is small. A more rigorous reflection model is required to improve a measurement accuracy lower than $150 \mathrm{~nm}$. In conclusion, the most important advantage of the proposed technique is the elimination of the ambiguity in determining the thickness and surface profile. By separating measurement of thickness and surface profile, the proposed method effectively eliminated the ambiguity of thickness determination.

\section{ACKNOWLEDGMENT}

This work was supported by the SNUPrecision and also supported in part by the Institute of Advanced Machinery and Design at Seoul National University.

\section{REFERENCES}

1. H. G. Tompkins and W. A. McGahan, Spectroscopic Ellipsometry and Reflectometry: A User's Guide (Wiley, New York, USA, 1999), pp. 222-224.

2. H. Fujiwara, Spectroscopic Ellipsometry Principles and Applications (Wiley, New York, USA, 2007).

3. Y. M. Hwang, S. W. Yoon, J. H. Kim, S. Kim, and H. Pahk, "Thin-film thickness profile measurement using wavelet transform in wavelength-scanning interferometry," Opt. Lasers Eng. 46, 179-184 (2008).

4. S. W. Kim and G. H. Kim, "Thickness-profile measurement of transparent thin-film layers by white-light scanning interoferometry," Appl. Opt. 38, 5968-5973 (1999).

5. D. S. Kim and S. H. Kim, "Fast thickness profile measurement using a peak detection method based on an acoustooptic tunable filter," Meas. Sci. Technol. 13, L1-L5 (2002).

6. S. K. Debnath, J. You, and S. W. Kim, "Determination of film thickness and surface profile using reflectometry and spectrally resolved phase shifting interferometry," Int. J. Precis. Eng. Manuf. 10, 5-10 (2009).

7. P. Groot and L. Deck, "Surface profiling by analysis of white light interferograms in the spatial frequency domain," J. Mod. Opt. 42, 389-401 (1995).

8. P. Groot, X. Lega, J. Kramer, and M. Turzhitsky, "Determination of fringe order in white-light interference microscopy," Appl. Opt. 41, 4571-4578 (2002).

9. P. Groot, X. Lega, J. Kramer, and M. Turzhitsky, "Transparent film profiling and analysis by interference microscopy determination of fringe order in white-light interference microscopy," Proc. SPIE 7064, 70640I-1 (2008).

10. T. Jo, S. Kim, and H. Pahk, "3D measurement of TSVs using low numerical aperture white-light scanning interferometry," J. Opt. Soc. Korea 17, 317-322 (2013).

11. F. Gao, "Surface and thickness measurement of a transparent film using wavelength scanning interferometry," Opt. Express 20, 21450-21456 (2012).

12. P. Groot and X. Lega, "Transparent film profiling and analysis by interference microscopy," Proc. SPIE 7064, 794936 (2008).

13. D. Mansfield, "Extraction of film interface surfaces from scanning white light interferometry," Proc. SPIE 7101, 797978 (2008).

14. X. Jiang, K. Wang, F. Gao, and H. Muhamedsalih, "Fast surface measurement using wavelength scanning interferometry with compensation of environmental noise," Appl. Opt. 49, 2903-2909 (2010).

15. D. Kim, S. Kim, H. J. Kong, and Y. Lee, "Measurement of the thickness profile of a transparent thin film deposited upon a pattern structure with an acousto-optic tunablefilter," Opt. Lett. 27, 1893-1895 (2002).

16. Y. S. Ghim, A. Suratkar, and A. Davies, "Reflectometrybased wavelength scanning interferometry for thickness measurements of very thin wafers," Opt. Express 18, 65226529 (2010).

17. K. G. Larkin, "Efficient nonlinear algorithm for envelope detection in white light interferometry," J. Opt. Soc. Am. A 13, 832-843 (1996).

18. S. Kim, J. Kim, and H. Pahk, "Fringe-order determination method in white-light phase-shifting interferometry for the 
compensation of the phase delay and the suppression of excessive phase unwrapping," J. Opt. Soc. Korea 17, 415422 (2013).

19. D. Yoon, T. Kim, M. Kim, and H. Pahk, "Unambiguous 3D surface measurement method for a micro-Fresnel lensshaped lenticular lens based on a transmissive interferometer,"
J. Opt. Soc. Korea 18, 37-44 (2014).

20. H. Kang, J. Lim, P. Peranantham, and C. HwangBo, "Determination of optical constants of thin films in extreme ultraviolet wavelength region by an indirect optical method," J. Opt. Soc. Korea 17, 38-43 (2013). 